\title{
4. Ueber wechselseitige Diffusion von Electrolyten in verdünnten wässerigen Lösungen, insbesondere über Diffusion gegen das Concentrationsgefülle; von U. Behn.
}

Auf Grund der van't Hoff'schen Anschauungen über die Natur der Lösungen hat Hr. Nernst ${ }^{1}$ ) eine Theorie der Hydrodiffusion entwickelt, die einen Einblick in die Mechanik derselben eröffnete und die sich in dem vorhandenen Zahlenmaterial von Diffussionsbeobachtungen, soweit es sich bei den Fehlern dieser und den vereinfachenden Annahmen der Theorie erwarten liess, aufs beste bestätigte. Jedoch waren die Betrachtungen zunächst beschränkt auf eine einzige Lösung. Hr. Planck ${ }^{2}$ ) zeigte dann, wie man beim Vorhandensein einer gemeinschaftlichen Grenzfläche ${ }^{3}$ ), also für den Fall zweier Lösungen von beliebig vielen binären Electrolyten vorzugehen habe. Dadurch wurde es möglich, die Theorie an Versuchen zu prüfen, bei denen zwei Electrolyte wechselseitig diffundiren; während vorher nur die Diffusion in das reine Lösungsmittel durch Vergleich mit den von der Theorie geforderten Werthen Interesse erregte und, von vereinzelten Versuchen abgesehen, in der That auch allein Gegenstand von Beobachtungen gewesen war. Es boten sich nun hier verschiedene Probleme, die rechnerisch verfolgt werden konnten, unter denen eins den praktischen Schwierigkeiten, die aus der grossen Langsamkeit der Hydrodiffusionsvorgänge erwachsen, am ehesten zu entgehen schien.

Es war das die Frage nach dem Verhältniss der gleichzeitig durch die Grenzschicht diffundirenden Mengen von Ionen, wenn zwei homogene verdünnte Lösungen von je einem oder mehreren binären Electrolyten aneinander grenzen. Um die

1) W. Nernst, Zeitschr. f. phys. Chem. 2. p. 613. 1888; 4. p. 129. 1889 ; vgl. auch L. Planck, Wied. Ann. 39. p. 161. 1890.

2) M. Planck, Wied. Ann. 40. p. 561. 1890.

3) Die äusseren Grenzen der Lösungen kommen, wenn diese nur hinreichend gross gewählt sind nicht in Betracht. 
beiden Lösungen während des Versuchs annähernd homogen zu erbalten, musste ihre gemeinschaftliche Grenzfläche klein sein gegen ihre Querschnitte und ihre Volumina und die Versuche durften nur solange dauern, als man noch von einer bestimmten Grenzschicht sprechen konnte, d. h. solange das Concentrationsgefälle beschränkt blieb auf eine Schicht, die klein war gegen die sonstigen Dimensionen der Lösungen und solange es zulässig war, die diffundirten Mengen gegen die ursprüuglich in den Lösungen vorhandenen $z \mathbf{u}$ vernachlässigen. Jedoch wurde der Analyse wegen diese Grenze erst bei mehreren Procent gezogen und es betrug die Versuchsdauer bei den Dimensionen der verwendeten Diffusionsgefässe immerhin 1-2 Wochen.

Es sei gleich vorweggenommen, dass die Versuchsresultate mit den von der Theorie geforderten quantitativ schlecht übereinstimmen und $z$ war wahrscheinlich deshalb, weil es nicht gelang, Strömungen hinreichend zu beseitigen.

Nehmen wir an, dass die beiden Lösungen sich gar nicht durch Diffusion, sondern nur durch Strömungen mischten, so müsste bei gleicher Concentration beider das Verhältniss der durch die Grenzschicht gewanderten Mengen offenbar stets 1 betragen. Wirken Strömungen also neben der Diffusion, so werden erstere die Verhältnisszahl der 1 immer nähern.

Dies zeigen nun auch einige Resultate über Diffusion zweier gleichconcentrirter Lösungen ineinander, die nur kurz erwähnt werden sollen. Dann sei es mir erlaubt, Versuche mitzutheilen, bei denen schon die qualitative Uebereinstimmung mit der Theorie Interesse zu verdienen scheint, weil sie in einem bestimmten Fall die Theorie bestätigen, in dem diese Diffusion gegen das Concentrationsgefälle vorausgesagt hat.

Der Weg, den Hr. Planck ${ }^{1}$ ) eingeschlagen hat, um das Verhältniss der gleichzeitig durch die Grenzfläche wandernden Ionen zu berechnen, möge hier nur kurz skizzirt werden.

Die Anzahl der durch die Grenzschicht ${ }^{2}$ ) gehenden Kationen einer bestimmten Art ist

1) Planck, Wied. Ann. 40. p. $561 \mathrm{ff}$.

2) Um einen Sprung in den Concentrationen zu vermeiden, wird eine Grenzschicht von sehr geringer Dicke angenommen. 
Anzahl $=$ Beweglichkeit $\times$ Lraft $\times$ Zeit $\times$ Concentration $\times$ Fläche

$$
\mathfrak{A}^{\prime}=-u^{\prime}\left(\varepsilon \frac{\partial \varphi}{\partial x}+\frac{R \vartheta}{c^{\prime}} \cdot \frac{\partial c^{\prime}}{\partial x}\right) d \boldsymbol{t} \cdot c^{\prime} \cdot d y d z,
$$

wo bedeuten: $u^{\prime}$ Beweglichkeit der Kationen erster Art (ebenso $v^{\prime}, v^{\prime \prime}$ etc. für die Anionen), $c^{\prime}$ ihre Anzahl in der Volumeneinheit ${ }^{1}$ ), (ebenso $\bar{c}^{\prime}, \bar{c}^{\prime \prime}$ für die Anionen) $\varepsilon$ die Ladung eines Ions, $\varphi$ das electrostatische Potential, $R$ den osmotischen Druck bei der Partialconcentration 1 und der Temperatur 0, $\vartheta$ die absolute Temperatur und $t$ die Zeit. Nun hat man sogleich in $\mathfrak{A}^{\prime}: \mathfrak{A}^{\prime \prime}: \ldots$ die gesuchten Verhältnisse; jedoch kennen wir $\partial \varphi / \partial x$ noch nicht. Nach Ablauf von a priori möglichen, aber sehr labilen Zuständen werden die Differentialquotienten der Concentrationen nach der Zeit klein gegen die räumlichen. Die hierdurch ermöglichten Vereinfachungen führen zu der Gleichung

$$
\frac{\partial \varphi}{\partial x}=\underset{\varepsilon\left[x\left(c_{2}-c_{1}\right)+c_{1} \delta\right] \log \frac{\left(c_{2}-c_{2}\right) R}{c_{1}}}{\cdots},
$$

worin die $c$ ohne oberen Index die Gesammtconcentrationen der Lösungen, die Hülfsgrösse

$$
\xi=\left(\frac{c_{2}}{e_{1}}\right)^{\frac{(A-B) \delta}{2\left(c_{2}-c_{1}\right) R \vartheta}}
$$

$\delta$ Dicke der Grenzschicht, $A$ und $B$ Integrationsconstanten sind (vgl. l. c.); und zur Berechnung von $\xi$ ergiebt sich (l. c. p. 566,567$)$

wo

$$
\frac{\xi U_{2}-U_{1}}{V_{2}-\xi V_{1}}=\frac{\log _{c_{1}}^{c_{2}}-\log \xi}{\log _{c_{1}}^{c_{2}}+\log \xi} \cdot \frac{\xi c_{2}-c_{1}}{c_{2}-\xi c_{1}}
$$

$$
\begin{aligned}
& U_{1}=u_{1}^{\prime} c_{1}^{\prime}+u_{1}^{\prime \prime} c_{1}^{\prime \prime}+\ldots \\
& V_{1}=v_{1}^{\prime} c_{1}^{\prime}+v^{\prime \prime} c^{\prime \prime}+\ldots
\end{aligned}
$$

Setzt man nun (8) in die Gleichung für $\mathfrak{U}^{\prime}$ ein, so erhält man

$$
A^{\prime}=\frac{\mathfrak{H}^{\prime}}{-u^{\prime} d y d z d t}=\frac{e^{\prime}\left(c_{2}-c_{1}\right) R \vartheta \log \xi}{\left[x\left(c_{2}-c_{1}\right)+c_{1} \delta\right] \log \frac{c_{2}}{c_{1}}}+R \vartheta \frac{\partial c^{\prime}}{\partial x}
$$

1) Als Volumeneinheit ist bei den späteren Zahlenangaben statt des $\mathrm{cm}^{3}$ das Liter gewählt. Dadurch wird nichts in den Gleichungen geändert; man hat aber so die Concentrationen direct auf Normallösungen bezogen.

2) Die Numerirung ist die l. c. von Hrn. Planck gebrauchte. 
Diese in Bezug auf $c^{\prime}$ lineare Differentialgleichung zwischen den Variablen $c^{\prime}$ und $x$, integrirt, giebt

$$
\begin{aligned}
c^{\prime} & =\frac{A^{\prime}}{R \frac{y}{y}} \cdot \frac{x\left(c_{2}-c_{1}\right) \log \frac{e_{2}}{e_{1}}+c_{1} \log \frac{e_{2}}{c_{1}} \delta}{\left(c_{2}-c_{1}\right)\left(\log \xi+\log \frac{c_{2}}{c_{1}}\right)} \\
& +\left\{\left(c_{2}-c_{1}\right) \log _{c_{1}}^{c_{2}} \cdot x+c_{1} \log _{c_{1}}^{c_{2}} \delta\right\}^{-\log \frac{\log _{c_{2}}}{c_{1}} \text {. const. }}
\end{aligned}
$$

und wenn man die Integrationsconstante eliminirt unter Berïcksichtigung, dass für

$$
x=o c^{\prime}=c_{1}^{\prime} \text { und für } x=\partial^{\prime} c^{\prime}=c_{2}^{\prime}
$$

ist, so erhält man

$$
t^{\prime}=\left\{R \vartheta \cdot \begin{array}{c}
c_{2}-c_{1} \\
c_{2} \xi-c_{1}
\end{array}\left[\begin{array}{c}
\log \xi+\log e_{2} \\
c_{1} \\
c_{1} c_{2}
\end{array}\right]\left(c_{2} \xi-c_{1}\right)\right\}
$$

und wenn man die entsprechenden $A^{\prime \prime}$ etc. bildet und durch $-u^{\prime} d y d z d t$ dividirt, so erhält man in

$$
\mathfrak{A}^{\prime}: \mathfrak{l}^{\prime \prime}: \ldots=\left(c_{1}^{\prime}-c_{2}^{\prime} \xi\right) u^{\prime}:\left(c_{1}^{\prime \prime}-c_{2}^{\prime \prime} \xi\right) u^{\prime \prime}: \ldots
$$

die gesuchten Verhältnisse; und hierin sind die $\mathfrak{A}^{\prime}, \mathfrak{A}^{\prime \prime}, \ldots$ einzeln von gleichem Vorzeichen wie die entsprechenden Glieder der Proportion. Was experimentell auf dem eingeschlagenen Wege bestimmt werden kann, sind eben auch nur Verhältnisse von diffundirten Ionenmengen. Um absolute Diffusionscoefficienten zu erhalten, müssten wir $\delta$ kennen.

\section{Verguchsanordnung.}

Die für die Versuche verwandten Diffusionsgefässe hatten alle annähernd gleiche Form und gleiche Dimensionen; sie waren folgendermaassen hergestellt: Von einer $3 / 4$ l-Flasche $B$ (vgl. Fig 1 auf folgender Seite) mit Tubus am Boden wurde der Hals abgesprengt und die so entstandene ringförmige Bruchstelle eben geschliffen. Auf dieselbe wurde dann eine ca. $2 \mathrm{~mm}$ dicke Glasplatte $d$, in die ein Loch von $5 \mathrm{~mm}$ Durchmesser gebohrt war, centrisch aufgekittet. Mittels einer kleinen Schiebervorrichtung konnte ein Deckgläschen $c$ über das Loch geschoben werden. Ueber den oberen Theil der 
Flasche $B$ wurde dann die etwas weitere Flasche $A$, deren Boden abgesprengt war, so übergekittet, dass $A$ dem Volumen $B$ ungefähr gleich wurde. In die Wandung von $A$ war vorher in geeigneter Höhe ein Loch gebohrt, das mit einem angekitteten Kork $a$ als Stopfbüchse verschlossen, einen Platindraht $b$ durchliess, der seinerseits am Ende an das Deckgläschen $c$ gekittet, dieses hin- und herzuschieben erlaubte. Als Kitt wurde theils die bekannteWachs-Kolophonium-Mischung, theils Chatterton compound benutzt.

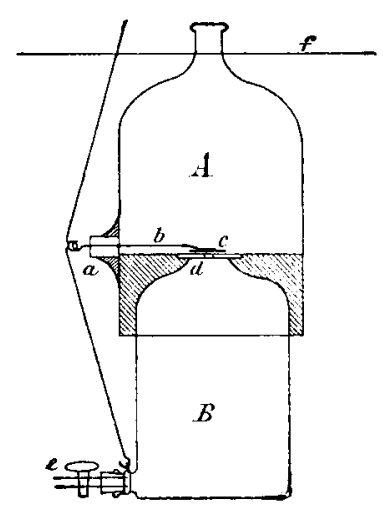

Fig. 1.

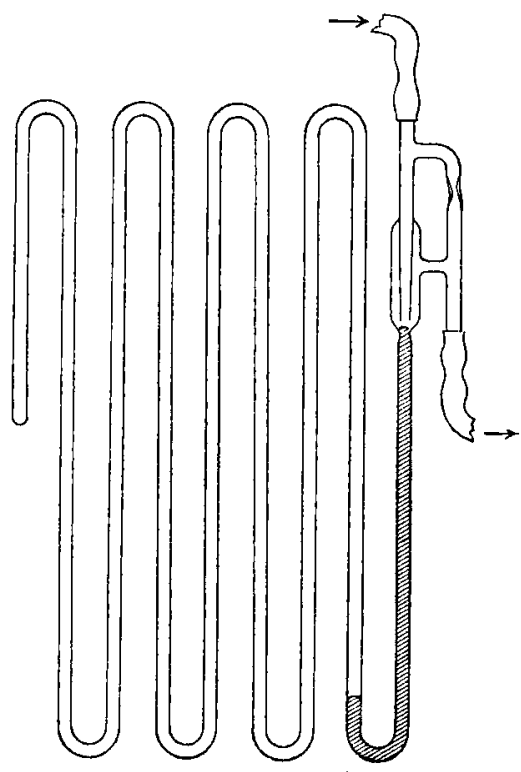

Fig. 2.

Die Füllung von $B$ geschah durch das Hahnrohr $e$ bei offenem Schieber, bis die (schwerere) Lösung den Boden von $A$ bedeckte. Nachdem man sich überzeugt, dass keine Luftblasen mehr in $B$ waren bez. diese entfernt hatte, wurde $A$ bei geschlossenem $c$ mit der anderen Lösung ausgespült und gefüllt.

Dann wurde das Diffusionsgefäss bis $f$ in ein Wasserbad eingesenkt, dessen Boden von einer horizontalen Glasplatte gebildet war, und nun, um eines guten Temperaturgleichgewichts sicher zu sein, mehrere Tage mit dem Beginn des Versuches gewartet. Das Badgefäss seinerseits war mit Filz auf allen Seiten bekleidet zum Schutz gegen die Temperaturschwankungen des Arbeitsraumes. Es war dies ein kleiner, durch Strohverkleidungen an Fenster und Thür möglichst 
gegen äussere Temperaturschwankungen geschützter Kellerraum des hiesigen Instituts. Immerbin zeigte ein Richard'scher' Thermograph, der mir von seiten der Physikalisch-Technischen Reichsanstalt freundlichst geliehen worden war, eine tägliche Periode. Bei den späteren Versuchen, die in den Wintermonaten ausgeführt sind, wurde der Raum mittels Gas-

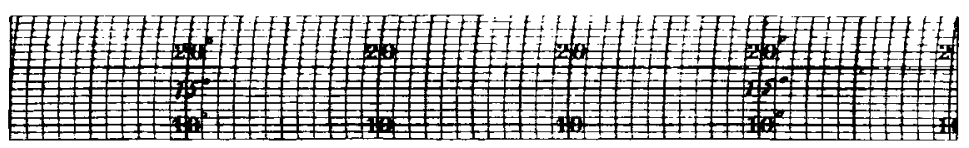

Fig. 3.

flammen und Thermoregulators ${ }^{1}$ ) (vgl. Fig. 2 p. 58) auf $18^{\prime \prime}$ gehalten ${ }^{2}$ ). Fig. 3 giebt ein beliebig herausgegriffenes Stück der rom Thermographen aufgezeichneten Temperaturcurve.

1) Derseibe war mit Petroleum gefüllt, das sich in einem $3.5 \mathrm{~m}$ langen aufgewundenen Rohr befand. Die regulirende Quecksilberkuppe bewegte sich gegen ein horizontal abgeschnittenes Rohr ohne Schlitz. Hr. Ostwald macht in seinem Hand- und Hülfsbuch zur Ausführung physiko-chemischer Messungen (p. 67) darauf aufmerksam, dass man durch letztere Einrichtung die Empfindlichkeit des Thermoregulators bedeutend steigern kann. Dass man sie bei den gebräuchlichen Constructionen nicht findet, liegt vielleicht an folgendem Umstand: Ist der Regulator in Thätigkeit, so versetzen die Gasblasen, die kurz vor völligem Abschluss noch über die Quecksilberoberfäche hin sich Bahn machen und in diesem Zustand befindet sich ein empfindlicher Regulator eigentlich immer - das Quecksilber in oscillirende Bewegung. Manchmal ist dieselbe (bei den gebräuchlichen Dimensionen) langsam : $2-3$ in der Sec.: meist aber so schnell, dass ein mässig hoher Ton entsteht. Die Flamme ist, indem sie entsprechende Zuckungen macht, in Gefahr zu verlöschen. Es liegt nun nabe, diesen Uebelstand durch Einschaltung eines grösseren Hohlraumes zwischen Flamme und Regulator zu beseitigen. Hierdurch erreichte ich auch eine gen ügende $\mathrm{Absch}$ wächung der Zuckungen; jedoch darf man die Flasche nicht zu gross wählen (etwa 1 l), weil sonst die Empfindlichkeit der Flamme gegen plötzliche kleine äussere Luftdruckänderungen von neuem die Gefahr des Verlöschens herbeifuhren würde. Durch Einschaltung von $60 \mathrm{l}$ z. B. direct vor der Flamme erreicht diese eine Empfindlichkeit, die der eines mittelgrossen Barovariometers (v. Hefiner-Alteneck, Wied. Ann. 57. p. 468. 1896) gleichkommt.

2) $\mathrm{Da}$ das Temperaturgefälle im Arbeitsraum in verticaler Richtung zunächst nicht unbeträchtlich war, wurde ein Ventilator verwandt, der, unausgesetzt in Betrieb, mittels einer passenden Einrichtung die wärmere Luft von der Decke her nach dem Fussboden beförderte. 
Ein gelegentlich vorkommender geringer Gang (bis $0,5^{\circ}$ ) ist wohl auf wechselnde Mengen Condensationsflüssigkeit über dem regulirenden Quecksilbermeniscus zurückzuführen. Fig. 3. (Die horizontale Entfernung von Zahl zu Zahl bedeutet 24 Stunden.)

\section{Wechselseitige Diffusion zweier binärer Electrolyte in gleichconcentrirten verdünnten Lösungen.}

Wenn ein langsamer Temperaturgang zu Strömungen naturgemäss wenig Veranlassung giebt und deshalb bei Diffusionsversuchen auch nicht so sehr zu fürchten ist, so sind andererseits kurzdauernde Temperaturschwankungen, selbst wenn sie sehr klein sind, eine schlimme Fehlerquelle. ${ }^{1}$ ) Dies gilt besonders beim Arbeiten mit verdünnten Lösungen, bei denen die Differenz der specifischen Gewichte nicht gross sein kann. Es war daher geboten, die Wahl von vornherein auf Electrolyte zu beschränken, deren specifische Gewichte bei gleichen, nicht zu grossen Concentrationen wenigstens einigermaassen differirten. Ausserdem durften sie chemisch nicht aufeinander wirken, sollten mit hinreichender Genauigkeit nebeneinander analysirbar sein und mussten endlich eine möglichst von 1 verschiedene Verhältnisszahl der gleichzeitig diffundirten Ionen ${ }^{2}$ ) erwarten lassen. Nach diesen Gesichtspunkten wurden zwei Combinationen $\mathrm{HCl}$, $\mathrm{LiCl}$ und $\mathrm{HNO}_{3}$, $\mathrm{AgNO}_{3}$, beide in $1 / 10$ normalen Lösungen gewählt.

Für gleiche Concentration auf beiden Seiten vereinfacht sich Gleichung (13) zu

da

$$
\xi=\frac{C_{1}+V_{2}^{\prime}}{V_{1}+\bar{U}_{2}^{\prime}}=\frac{u_{1}^{\prime} c_{1}^{\prime}+v_{2}^{\prime} c_{2}^{\prime}}{v_{1}^{\prime} c_{1}^{\prime}+u_{2}^{\prime \prime} c_{2}^{\prime \prime}}=\frac{\left.272+54{ }^{3}\right)}{54+24}=4,2,
$$

Dies ergiebt

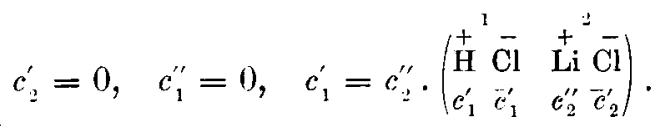

$$
\mathfrak{A}^{\prime}: \mathfrak{A}^{\prime \prime}=\left(c_{1}^{\prime}-c_{2}^{\prime} \xi\right) u^{\prime}:\left(c_{1}^{\prime \prime}-c_{2}^{\prime \prime} \xi\right) u^{\prime \prime}=c_{1}^{\prime} u^{\prime}:\left(-c_{2}^{\prime \prime} \xi\right) u^{\prime \prime}=2,7
$$

1) Vgl. Kawalki, Wied. Ann. 52. p. 177. 1894.

2) Im Folgenden ist stets die nur in grober Annäherung berechtigte Annahme gemacht, die verwendeten meist $1 / 10$ normalen Lösungen seien vollkommen dissociirt gewesen.

3) Bei Benutzung der von Hrn. Kohlrausch berechneten Beweglichkeiten für 0,1 normale Lösungen, vgl. Wied. Ann. 26. p. 214. 1885. 
für den ersten, $(\mathrm{HCl} / \mathrm{LiCl}), \mathfrak{A}^{\prime}: \mathfrak{A}^{\prime \prime}=1,8$ für den zweiten Fall $\left(\mathrm{HNO}_{3} / \mathrm{AgNO}_{3}\right)$. Gefunden wurde: 2,24 und 2,07, im Mittel 2,16 für $\mathrm{HCl}: \mathrm{LiCl}$; und $1,56,1,58,1,56,1,52,1,57,1,57$, 1,45, 1,69, im Mittel 1,56 für $\mathrm{HNO}_{3}: \mathrm{AgNO}_{3}$.

Die Analyse geschah durch Titrirung. Im ersten Fall wurde das $\mathrm{Cl}$ mittels $\mathrm{AgNO}_{3}$ im Ueberschuss gefällt und dieses gegen $\mathrm{NH}_{4} \mathrm{SCN}$ mit Eisenalaun als Indicator zurücktitrirt; die Säure gegen $\mathrm{KOH}$ mit Phenolphtaleïn; die Menge des $\mathrm{LiCl}$ also durch Subtraction gefunden.

Im zweiten Fall wurde das $\mathrm{Ag}$ gegen $\mathrm{NH}_{4} \mathrm{SCN}$ mit Eisenalaun bestimmt; die Säure gegen $\mathrm{KOH}$ mit Methylorange, da Phenolphtaleyn hier nicht verwendbar ist, wo wegen der Gegenwart von $\mathrm{AgNO}_{3}$ nach Neutralisation der Säure freies Alkali in der Lösung nicht bestehen kann. Die an und für sich schon geringere Empfindlichkeit der Methylorange wird übrigens durch die Gegenwart des Silbersalzes noch weiter herabgesetzt.

Trotzdem ist das Resultat der Titrirung hier zuverlässiger als bei der ersten Combination, da hier beide Electrolyte direct bestimmt werden kounten.

Man sieht, dass die gefundenen Werthe hinter den berechneten Verhältnisszahlen zurückbleiben, was sich, wie oben bemerkt, auf Strömungen zurückführen liesse.

\section{Diffusion gegen das Concentrationsgefälle.}

Das Verhältniss der gleichzeitig durch die Grenzschicht diffundirenden Kationen war:

$$
\mathfrak{A}^{\prime}: \mathfrak{A}^{\prime \prime}: \ldots=\left(c_{1}^{\prime}-c^{\prime} \xi \xi\right) u^{\prime}:\left(c_{1}^{\prime \prime}-c_{2}^{\prime \prime} \xi\right) u^{\prime \prime}: \ldots
$$

Hierin sind die Beweglichkeiten $u^{\prime}$. $u^{\prime \prime}$ etc. natürlich positiv, die Richtung der Diffusion hängt also von dem Vorzeichen der Klammer ab. Bei passend gewählten Concentrationen von Salzen, deren Ionen geeignete Beweglichkeiten haben, ist es nun möglich, dass $c_{i}^{\prime}-c_{2}^{\prime} \xi$ das entgegengesetzte Vorzeichen von $c_{1}^{\prime}-c_{2}^{\prime}$ hat; und zwar tritt dies offenbar dann ein, wenn die Einwirkung des osmotischen Druckes von den electrischen Kräften, die auf die Ionen ausgeübt werden. übercompensirt wird. In der That lassen sich Combinationen ron Electrolyten aufstellen, bei denen dies der Fall ist, die Diffusion also gegen das Concentrationsgefälle stattfinden muss. 
Z. B. wenn eine 1,1 normale Lösung (Index 1) von $\mathrm{NaC}_{2} \mathrm{H}_{3} \mathrm{O}_{2}$ und eine zweite (2) von $\mathrm{NaCl}$ (normal) und $\mathrm{LiCl}$ $(0,1$ normal) aneinander grenzen; dann ist

$$
\begin{aligned}
\xi=\frac{U_{1}+V_{2}^{\prime}}{V_{1}+U_{2}} & =\frac{u_{1}^{\prime} c_{1}^{\prime}+v_{2}^{\prime} \bar{c}_{2}^{\prime}}{v_{1}^{\prime \prime}} \frac{\bar{c}_{1}^{\prime \prime}+\left(u_{2}^{\prime} c_{2}^{\prime}+u_{2}^{\prime \prime} c_{2}^{\prime \prime}\right)}{} \\
& =\frac{32 \cdot 1,1+54 \cdot 1,1}{26 \cdot 1,1+32 \cdot 1,0+24 \cdot 0,1}=1,6 .
\end{aligned}
$$

Die Differenz der Concentrationen an Na-Ionen ist $c_{1}^{\prime}-c_{2}^{\prime}$ $=+0,1$. Dagegen $c_{1}^{\prime}-c_{2}^{\prime} \xi=-0,5$. Die Na-Ionen gehen also zur ersten (concentrirteren) Lösung.

Ein anderes Beispiel ist $\mathrm{AgNO}_{3}(<0,1$ normal) in der ersten, $\mathrm{AgNO}_{3}$ (0,1 normal) und $\mathrm{HNO}_{3}$ (normal) in der zweiten Lösung. Diese Combination ist für das Experiment geeigneter, denn einmal kommt darin kein so schwach dissociirter Electrolyt wie das essigsaure Natrium vor, und andererseits ist die Differenz der specifischen Gewichte dieser Lösungen bedeutend grösser. Hiermit sind denn auch einige Versuche angestellt, deren Resultate aus der folgenden Tabelle I ersichtlich sind.

In der 2.-5. Columne finden sich die Concentrationen bezogen auf Normal-Lösungen. Dann kommen die Werthe von $\xi$, die sich gemäss Formel (13) aus den Concentrationen und den Beweglichkeiten ${ }^{1}$ ) ergeben. In Columne 9-11 ist das Resultat der Titrirung, die wie oben ausgefübrt wurde, angegeben.

\begin{tabular}{|c|c|c|c|c|c|c|c|c|c|c|}
\hline 1 & 2 & 3 & \begin{tabular}{|l}
4 \\
\end{tabular} & $\tilde{5}$ & 6 & 7 & 8 & 9 & 10 & 11 \\
\hline Nr. & $\begin{array}{l}\text { Concer } \\
+-\overline{A g}_{3} \\
\hat{A g N}_{1}^{\prime}=\bar{c}_{1}^{\prime}\end{array}$ & $\mid \begin{array}{c}+ \\
\mathrm{Ag}^{\prime} \\
c_{2}^{\prime}\end{array}$ & $\begin{array}{c}(\mathrm{ab} \\
+ \\
\stackrel{\mathrm{H}}{ } \\
c_{2}^{\prime \prime}\end{array}$ & $\begin{array}{c}\text { ger.) } \\
\mathrm{NO}_{3} \\
e_{2}^{\prime}\end{array}$ & $\xi$ & $c_{1}^{\prime}-\iota_{2}^{\prime}$ & $c_{1}^{\prime}-c_{2}^{\prime} \xi$ & $\mid \begin{array}{c}\text { oben } \\
(1) \\
\text { fehlt }\end{array}$ & $\begin{array}{l}\text { mg-Ion } \\
\text { unten } \\
(2) \\
\text { mehr }\end{array}$ & Mittel \\
\hline 1 & 0,050 & $|0,100|$ & $\mid 1,01$ & 1,11 & 0,07 & $-0,050$ & $+0,043$ & 0,110 & 0,254 & 0,182 \\
\hline 2 & 0,049 & 0,098 & 1,01 & 1,11 & 0,07 & $-0,049$ & $+0,042$ & 0,149 & 0,319 & 0,234 \\
\hline 3 & 0,068 & 0,100 & 1,00 & 1,10 & 0,08 & $-0,032$ & $+0,060$ & 0,924 & 0,954 & 0,939 \\
\hline $\begin{array}{l}4 \\
5\end{array}$ & 0,090 & 0,098 & 0,99 & 1,09 & 0,10 & $-0,008$ & $+0,080 \mid$ & $\left\{\begin{array}{l}1,39 \\
1,65\end{array}\right.$ & $\begin{array}{l}1,21 \\
1,48\end{array}$ & $\begin{array}{l}1,30 \\
1,56\end{array}$ \\
\hline
\end{tabular}

Tabelle T.

1) Es sind auch hier die Beweglichkeiten für 0,1 normal benutzt. 
Quantitativ sind die Resultate allerdings recht weit davon entfernt eine Bestätigung der Theorie zu liefern; dazu war die Wahl der Concentrationen aber auch nicht geeignet. Immerhin mag noch folgende Zahlenzusammenstellung, dieselben 5 Versuche betreffend, hier Platz finden.

\begin{tabular}{|c|c|c|c|c|}
\hline 1 & 2 & 3 & 4 & 5 \\
\hline $\mathrm{Nr}$ & $\mathrm{Ag}$ & $\mathrm{H}$ & \multicolumn{2}{|c|}{ Verhältniss } \\
\hline & & & gef. & ber. \\
\hline 1 & 0,182 & 17,3 & $1: 95$ & $1: 11$ \\
\hline 2 & 0,234 & 19,9 & $1: 85$ & $1: 11$ \\
\hline 3 & 0,939 & 24,7 & $1: 26$ & $1: 9$ \\
\hline 4 & 1,30 & 17,2 & $1: 13$ & $1 \cdot 8$ \\
\hline 5 & 1,56 & 25,0 & $1: 16$ & \\
\hline
\end{tabular}

Columne 2 ist dieselbe wie 11 der ersten Tabelle. In 3 findet man die gleichzeitig übergegangenen Mengen an H-Ionen; unter 4 das Verhältniss beider, endlich in 5 dasselbe, berechnet. Man sieht, dass der Unterschied der Zahlen sub 4 und 5 besonders bei den ersten Versuchen sehr gross ist. Bei den späteren wird er dann kleiner.

Die Annahme, dass Strömungen die vorwiegende Ursache der Abweichungen sind, würde eine der relativen Grösse derselben entsprechende Erklärung liefern. Bei den letzten Versuchen der Tabelle ändern nämlich Strömungen, die etwa durch die Communicationsöffnung stattfinden, die Menge von Ag-Ionen auf beiden Seiten nur wenig gegeneinander, da die Differenz der Concentrationen $c_{1}^{\prime}$ und $c_{2}^{\prime}$ klein ist; stark aber wird die Concentration an $\mathrm{H}$-Ionen dadurch beeinflusst, da sie ursprünglich unten gleich 1 , oben gleich 0 ist; also in dem Bruch

$$
\frac{\text { Menge der durchgewanderten Ag-Ionen }}{\text { Menge der durchgewanderten H-Ionen }}
$$

nur der Divisor. Bei den ersten Versuchen aber ändert sich gleichzeitig der Dividend im entgegengesetzten Sinne nicht unerheblich, der Werth des Bruches also sehr stark. In der That zeigt eine Ueberschlagsrechnung, dass die Annahme einer in allen Versuchen fast gleichen infolge von Strömungen ạs- 
getauschten Menge von Lösung die sehr verschiedene $\mathrm{Ab}$ weichung der Resultate von den berechneten hinreichend erklären würde. Dass solche Strömungen durch die Erschütterungen begünstigt werden, denen selbst die Kellerräume des Instituts, das fast inmitten der Stadt liegt, im hohen Grade ausgesetzt sind, ist nicht ausgeschlossen.

Immerhin ist die Hauptursache der Strömungen wohl in Temperaturschwankungen zu suchen, und zwar bei den zuletzt beschriebenen Versuchen vielleicht weniger in solchen, die von aussen her eindrangen als vielmehr in Wärmetönungen, die im Innern der Gefässe durch die Diffusion selbst hervorgerufen wurden. Betrachten wir z. B. den 5. Versuch. Es sind in demselben $0,025\left(\mathrm{HNO}_{3}, 54 \mathrm{H}_{2} \mathrm{O}\right)$ auf $0,025\left(\mathrm{HNO}_{3}\right.$, aq. $)$ verdünnt. Die Wärmetönung bei der Lösung von $\mathrm{HNO}_{3}$ berechnet sich nach J. Thomsen ${ }^{1}$ ) nach der Formel

$$
\left.\left(\mathrm{HNO}_{3}, n \mathrm{H}_{2} \mathrm{O}\right)=\underset{n+1,737}{n} \cdot .89,74 K^{2}\right) \text {. }
$$

In unserm Falle also

$$
\begin{aligned}
0,025\left(\mathrm{HNO}_{3} .54 \mathrm{H}_{2} \mathrm{O}, \text { aq. }\right) & =0,025\left\{\left(\mathrm{HNO}_{3}, \text { aq. }\right)\right. \\
& \left.-\left(\mathrm{HNO}_{3}, 54 \mathrm{H}_{2} \mathrm{O}\right)\right\}=0,07 \mathrm{~K},
\end{aligned}
$$

eine Wärmemenge, die, selbst wenn sich ihre Entstehung auf 11 Tage vertheilt, zu nicht unerheblichen Strömungen Veranlassung geben kann.

Jedoch sind noch andere Umstände hier in Betracht zu ziehen. Es liegt in der Natur der Versuche, dass eine Anzahl von Bedingungen, die bei der Berechnung gemacht wurden, nur annähernd erfüllt sind; und $o b$ die eine oder die andere dieser Abweichungen eine wesentliche Fehlerquelle bildet, lässt sich schwer beurtheilen. Angenommen ist, dass die Electrolyte völlig dissociirt seien, dass auf beiden Seiten sich reine Lösungen befinden, $d . h$. dass auch bei Beendigung des Versuches die diffundirten Mengen verschwindend klein seien, dass also während des ganzen Versuches das Concentrationsgefälle auf die Grenzschicht beschränkt sei, die von sehr kleiner Dicke angenommen ist. Dies alles lässt sich ja nur annähernd erreichen.

1) J. Thomsen, Thermochem. Untersuchungen. 3. p. $s$ ff.

2) $\mathrm{K}=100$ cal.

don. d. Phys, u, Chems. N. F. 62. 
Wie dick in Wirklichkeit die Grenzschicht nach Ablauf der halben Versuchsdauer etwa ist, lässt sich übrigens der Grössenordnung nach bestimmen. Der Diffusionscoefficient ist für $\mathrm{HNO}_{3}$ in wässeriger Lösung, wenn kein anderer Electrolyt zugegen ist, bei $18^{\circ}$ etwa $2,3 .^{1}$ ) Nun bleibt der Diffusionscoefficient bei Anwesenheit eines anderen Electrolyten ja nicht unverändert; vielmehr weiss man, dass z. B. bei der Diffusion zweier Electrolyte aus wässeriger Lösung in reines Wasser grosse Coefficienten noch grösser werden und umgekehrt. Angenommen aber, dass eine eventuelle Veränderung des Diffusionscoefficienten in unserem Fall zu vernachlässigen wäre ${ }^{2}$ ), so hätten wir nach dem Fick'schen Gesetz, das sich ja auch aus der Nernst'schen Theorie ergiebt,

$$
S_{a}=S_{k}=k \cdot q \cdot t \cdot \frac{d c}{d x} .
$$

$d c$ ist hierin die (räumliche) Concentrationsänderung, wenn man von der einen Lösung zur anderen geht, oder mit anderen Worten die bekannte Concentrationsdifferenz der beiden Lösungen; $d x$ die Strecke, in der die Concentrationsänderung von einem Grenzwerth zum anderen vor sich geht, die gesuchte Dicke $\delta$ der Grenzschicht.

$$
d x=\frac{k \cdot q \cdot t \cdot d e}{S}=\frac{2,3 \cdot 0,2 \cdot 9,4 \cdot 0,001}{0,021}=0,2 \mathrm{~cm},
$$

wenn man die Mittel aus Versuch $1-5$ einsetzt: $q=0,2 \mathrm{~cm}^{2}$, $t=9,4$ Tage, $d c=0,001 \mathrm{~g}$-Ion und $S=0,021 \mathrm{~g}$-Ion.

Berlin, Physik. Institut d. Univ., Mai 1897.

Nachtrag.

Während diese Arbeit abgeschlossen wurde, erschien eine Arbeit des Hrn. Tammann ${ }^{3}$ ): Ueber den Theilungscoefficienten und abnorme Diffusion. In derselben wird eine grössere Anzahl Versuche über Diffusion gegen das Concentrationsgefälle

1) Vgl. Scheffer, Zeitschr. f. phys. Chemie 2. p. 395.1888.

2) Ein diesbezüglicher Versuch ergab eine geringe Abnahme des Coefficienten für die Diffusion in $\mathrm{AgNO}_{3}$-Lösung.

3) Tammann, Zeitschr. f. phys. Chemie. 22. p. 481. 
beschrieben, auch eine Arbeit des Hrn. Abeggi ${ }^{\mathrm{I}}$ ) citirt, die mir entgangen war, und in der ebenfalls mehrere Fälle von Diffusion gegen das Concentrationsgefälle mitgetheilt werden. Der wesentliche Unterschied zwischen diesen und den obigen von Hrn. Planck theoretisch vorausgesagten, liegt wohl darin, dass bei jenen Versuchen Diffusion von einem Lösungsmittel in ein anderes stattfindet. Auch scheint dort (wenigstens bei den Versuchen des Hrn. Abegg) Abweichung von dem van 't Hoff'schen Gesetz über die Proportionalität von Concentration und osmotischem Druck maassgebend zu sein, während es sich bei mir ausschliesslich um verdünnte wässerige Lösungen handelt, die Gültigkeit jenes Gesetzes also vorausgesetzt ist. Der Vorgang in den hier mitgetheilten Versuchen ist an das Vorhandensein von Ionen gebunden, da es erst die zu dem osmotischen Druck hinzutretenden electrischen Zusatzkräfte sind, die hier die Diffusion gegen das Concentrationsgefälle hervorrufen.

1) Abegg, Zeitschr. f. phys. Chemie. 11. p. 257. 1893.

(Eingegangen 29. Mai 1897.) 